\title{
Radiologic and histologic findings in Sjögren's sensory neuronopathy
}

Achados radiológicos e histológicos da neuronopatia sensitiva da síndrome de Sjögren

Ronnyson Susano GRATIVVOL' , Wagner Cid Palmeira CAVALCANTE', Lais Maria Gomes de Brito VENTURA', Vitor Marques CALDAS', Leandro Tavares LUCATO², Silvia Vanessa LOURENCCO³, Carlos Otto HEISE ${ }^{1}$; Ricardo NITRINI ${ }^{1}$

A 27-year-old woman presented with a two-year history of asymmetric paresthesia in her hands and feet. Neurological examination revealed global areflexia, marked loss of position sense and normal motor strength in all limbs. Nerve conduction studies showed absence of sensory potentials with no abnormalities in electromyography. These findings were compatible with a diagnosis of sensory neuronopathy. Spine MRI demonstrated hyperintensity in the posterior columns (Figures A and B). Salivary gland biopsy (Figure C) was diagnostic for Sjögren's syndrome. Sensory neuronopathy is a rare type of peripheral neuropathy, which can occur among Sjögren's syndrome patients and usually antedates the diagnosis of Sjögren's syndrome ${ }^{1,2}$.

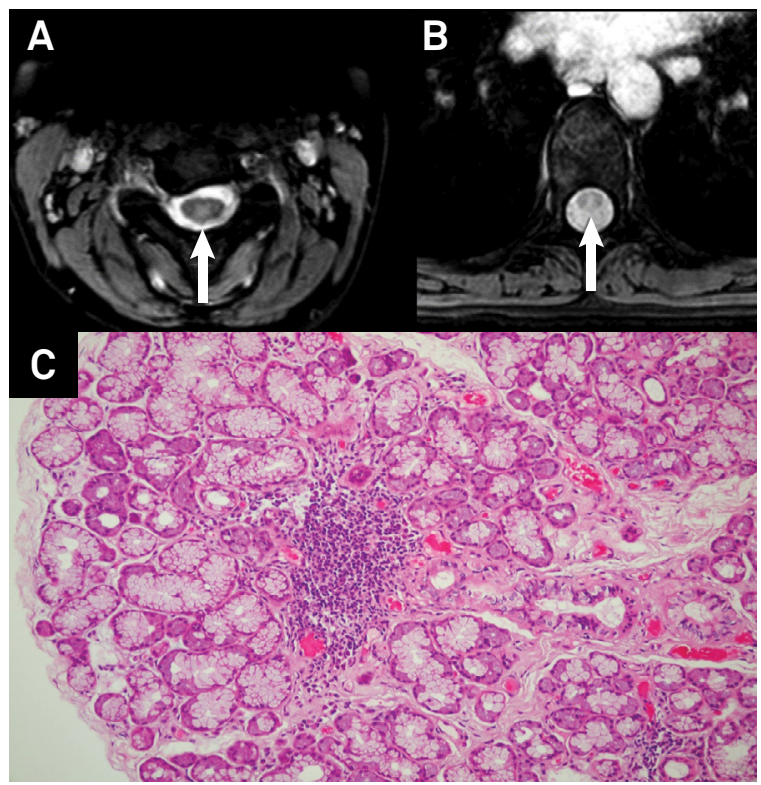

Figure. Axial T2 gradient-echo images (A and B) show hyperintensity involving the posterior columns of the spinal cord at cervical (A) and thoracic (B) levels (arrows). Histopathology images (C) shows lymphocytic infiltrate with complete destruction of the excretory duct. Acinic serous metaplasia and thrombi in small blood vessels are also seen (H\&E, original magnification X200).

\section{References}

Gwathmey KG. Sensory neuronopathies. Muscle Nerve 2016

Jan;53(1):8-19. https://doi.org/10.1002/mus.24943
2. Martinez ARM, Nunes MB, Nucci A, França Junior MC. Sensory neuronopathy and autoimmune diseases. Autoimmune Dis. 2012;2012: 873587. https://doi.org/10.1155/2012/873587

\footnotetext{
'Universidade de São Paulo, Faculdade de Medicina, Hospital das Clínicas, Departamento de Neurologia, São Paulo SP, Brasil; 2Universidade de São Paulo, Faculdade de Medicina, Hospital das Clínicas, Instituto de Radiologia, São Paulo SP, Brasil; ${ }^{3}$ Universidade de São Paulo, Faculdade de Medicina, Hospital das Clínicas, Departamento de Dermatologia, São Paulo SP, Brasil. Ronnyson Susano Grativvol (iD) https://orcid.org/0000-0001-8136-900X; Wagner Cid Palmeira Cavalcante (iD https://orcid.org/0000-0003-1453-900X; Lais Maria Gomes de Brito Ventura (iD https://orcid.org/0000-0002-8397-3523; Vitor Marques Caldas iD https://orcid.org/0000-0003-2839-3611; Leandro Tavares Lucato (iD https://orcid.org/0000-0001-9181-5245; Silvia Vanessa Lourenço iD https://orcid.org/0000-0002-6704-8549; Carlos Otto Heise (iD) https://orcid.org/0000-0003-3956-3073; Ricardo Nitrini (iD) https://orcid.org/0000-0002-5721-1525

Correspondence: Ronnyson Susano Grativvol; Departamento de Neurologia do HCFMUSP; Av. Dr. Enéas de Carvalho Aguiar, 255 / $5^{\circ}$ andar; $05403-001$ São Paulo SP, Brasil; E-mail: roninsg@hotmail.com

Conflict of interest: There is no conflict of interest to declare.

Received 01 June 2019; Accepted 16 July 2019.
} 